\title{
International Space Station Water Balance Operations
}

\author{
Barry Tobias $^{1}$ and John D. Garr II ${ }^{1}$. \\ NASA Lyndon B. Johnson Space Center, Houston,TX, 77058 \\ Meghan Erne ${ }^{1}$ \\ Barrios Technology, Houston, TX, 77058
}

In November 2008, the Water Regenerative System racks were launched aboard Space Shuttle flight, STS126 (ULF2) and installed and activated on the International Space Station (ISS). These racks, consisting of the Water Processor Assembly (WPA) and Urine Processor Assembly (UPA), completed the installation of the Regenerative (Regen) Environmental Control and Life Support Systems (ECLSS), which includes the Oxygen Generation Assembly (OGA) that was launched 2 years prior. With the onset of active water management on the US segment of the ISS, a new operational concept was required, that of "water balance". In November of 2010, the Sabatier system, which converts $\mathrm{H} 2$ and $\mathrm{CO} 2$ into water and methane, was brought on line. The Regen ECLSS systems accept condensation from the atmosphere, urine from crew, and processes that fluid via various means into potable water, which is used for crew drinking, building up skip-cycle water inventory, and water for electrolysis to produce oxygen. Specification (spec) rates of crew urine output, condensate output, $\mathrm{O} 2$ requirements, toilet flush water, and drinking needs are well documented and used as the best guess planning rates when Regen ECLSS came online. Spec rates are useful in long term planning, however, daily or weekly rates are dependent upon a number of variables. The constantly changing rates created a new challenge for the ECLSS flight controllers, who are responsible for operating the ECLSS systems onboard ISS from Mission Control in Houston. This paper reviews the various inputs to water planning, rate changes, and dynamic events, including but not limited to: crew personnel makeup, Regen ECLSS system operability, vehicle traffic, water storage availability, and Carbon Dioxide Removal Assembly (CDRA), Sabatier, and OGA capability. Along with the inputs that change the various rates, the paper will review the different systems, their constraints, and finally the operational challenges and means by which flight controllers manage this new concept of "water balance."

\section{Introduction}

$\mathrm{T}$ he International Space Station (ISS) United States Orbital Segment (USOS) Regenerative Environmental Control and Life Support Systems (ECLSS) recovers water from condensate and urine, provides potable water and oxygen for the crew, and supplys water to other systems. One of these systems, the Oxygen Generation Assembly (OGA), was launched onboard STS-121 (ULF1.1) in July of 2005. However, the rest of the Regen ECLSS systems did not reach orbit until November 2008 onboard STS-126 (ULF2). These new systems included the Urine Processor Assembly (UPA), Water Processor Assembly (WPA), Potable Water Dispenser (PWD) and Waste and Hygiene Compartment (WHC). The last system to arrive on ISS was the Sabatier, which launched on STS-131 (19A) in April of 2010. Although these systems are independent boxes, they are very much integrated as they are all variables in the overall water management system that is known as "water balance" by the ISS ECLSS flight control team.

\section{A. Waste Hygiene Compartment}

The WHC is the USOS toilet. It consists of a Russian ACY outfitted with extra sensors to help automate flush tank fills. The WHC consists of a ЕДВ-СВ which is a 22 liter container of non-iodinated water used for flush. Upon crew use, crew urine is mixed with a small amount of pretreat and flush water and is stored in another ЕДВ called a ЕДВ-У or passed through a jumper that feeds into the UPA.

\footnotetext{
${ }^{1}$ ISS ECLSS Officer, DI52/MOD ECLSS, NASA, Lyndon B. Johnson Space Center, Houston, TX, 77058.
} 


\section{B. Urine Processor Assembly}

The UPA accepts pretreated urine from the WHC (or pumped in from an ЕДВ-У). The urine is stored in the Wastewater Storage Tank Assembly (WSTA) until it is ready for batch processing, at which point it then passes through a vapor compression distillation process where distillate is separated from the pretreated urine. The distillate that is reclaimed from the pretreated urine is passed through to the WPA. The concentrated brine fluid is collected within a Recycled Tank Filter Assembly (RFTA), which is replaced upon reaching a certain throughput. This replacement schedule is such that the fluid does not become overly concentrated, thereby preventing precipitates from coming out of solution and ultimately failing the UPA.

\section{Water Processor Assembly}

The WPA accepts fluid from five sources. The first is the UPA's distillate output. The second source is from condensate collected from the ISS atmosphere. This condensate is water collected from human sweat and other sources of evaporated water (towels, food, wet trash, etc.) The third source is from Sabatier, which will be discussed below. The fourth is water pumped into the waste water bus/tank from an external storage source, such as a СWС or ЕДВ. The fifth and final source is water rejected by the OGA. The WPA processes this water through a variety of filters and a catalytic oxidizer. The processed potable water is then pumped into a potable bus, which feeds the OGA, PWD, and fills the WHC's ЕДВ-CB when required.

\section{Potable Water Dispenser}

The PWD is the crew's galley. The PWD can dispense ambient or hot water and dispenses the water into drink bags or food containers to rehydrate food. The PWD strips all biocide from this water prior to dispensing it for crew consumption. Additionally, the PWD has an Auxiliary (AUX) port which can be used to fill CWC-I with iodinated water.

\section{E. Oxygen Generation Assembly}

The OGA pulls water from the potable water bus and via electrolysis, creates oxygen and hydrogen. The oxygen is vented into the cabin for crew metabolic use, whereas the hydrogen is either vented overboard or passed along to the Sabatier.

\section{F. Sabatier}

The Sabatier system accepts hydrogen from OGA's electrolysis process and CO2 from the Node 3 Carbon Dioxide Removal Assembly (CDRA). The hydrogen and carbon dioxide are combined and produces water and methane gas. The product water is passed on to WPA for processing, and the methane is vented overboard.

\section{G. Lab Condensate Tank}

The Lab condensate tank was originally the prime storage tank for USOS collected condensate. However, now that the WPA is onboard, this tank is used as a backup to collect condensate at times when WPA's Waste Water (WW) tank is not available. Water can only be retrieved from the tank by offloading into CWCs (see next paragraph) or (with significant planning and coordination) it can be vented overboard. Both operations require crew time.

\section{H. Contingency Water Container \& Contingency Water Container - Iodine}

There are two main fluid containers that are used in support of the USOS water systems on ISS. The first is the Contingency Water Container (CWC). It is a soft goods container and can be used to hold most types of water: potable, technical, condensate, and waste. There is an alternate CWC, called the CWC-Iodine, that can hold the same types of water as the CWC, as well as being certified to hold iodinated water. Surplus water from the USOS 
water system can be offloaded into them, or the water being stowed in these bags can be used to supplement the water system as required by water balance needs.

There are two ways in which water can be put back into the USOS water system from a CWC. The first is via a condensate pump (peristaltic pump) that transfers water into the WPA WW tank. Second, a T-hose allows iodinated water to be put into the WPA Water Storage (WS) tank. As the water storage tank is sub ambient, the water is drawn in to the tank by bellow forces.

\section{I. ЕДВ}

The other main fluid container is the Russian ЕДВ. Тhe ЕДВ is a soft good container inside a hard shell. While these can also hold different types of water, it is mostly used on the U.S. segment for pre-treated urine (called a ЕДВ-У). As required, the WНС can be configured to feed pre-treated urine into a ЕДВ instead of directly into the UPA. As needed, the ЕДВs, full of pre-treated urine, can be put back into the UPA WSTA using a compressor or emptied into a Progress Rodnik tank. The Russian Program controls the loading of the Rodnik tanks and the U.S. ЕДВs are second priority to Russian ЕДВs. Therefore, there isn't always room to offload the containers. ЕДВs have a 90 day lifetime and if they cannot be emptied into UPA or into a Rodnik tank, they can't be reused and must be thrown away full.

\section{J. Specification Rates}

When the Regen ECLSS systems were designed, a certain input/output had to be assumed. These came from spec rates which the ISS program has documented as the expected crew metabolic rates, reference Figure 1.
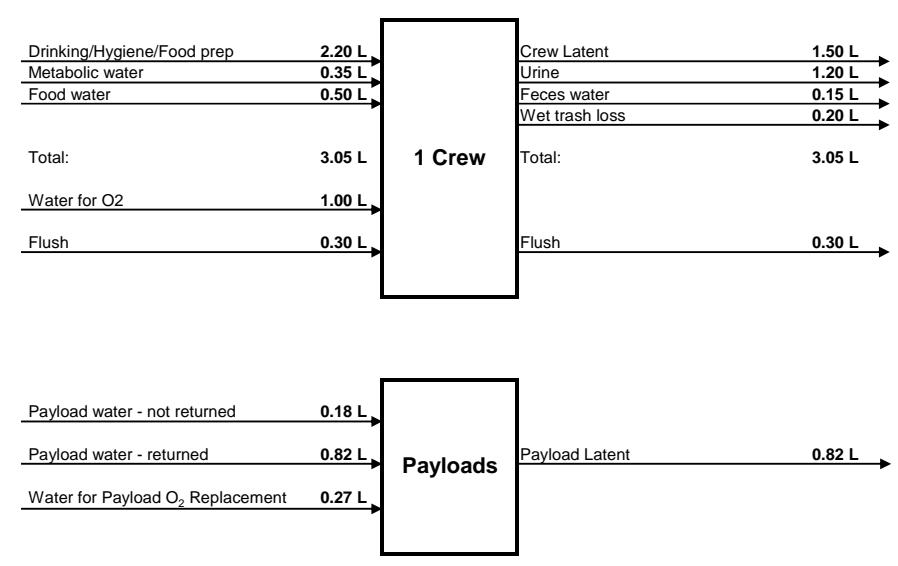

\section{Figure 1: Daily Water Planning Rates}

Over a long period of time, the ISS crew metabolic rates have been close to accurate. Over multiple increments (6 month stays of a crew of 3 persons), actual rates match those of the documented spec rates. This accuracy has been vital in terms of insuring the proper consumables are onboard, or whether or not water or oxygen needs to be manifested on upcoming vehicles to supplement the onboard inventory.

\section{K. Water Balance}

The Regen ECLSS systems are very much a closed loop system, in that crews' production of sweat and urine end up being processed into potable water, flush water, and oxygen, which then once again becomes crews' sweat and urine for reprocessing. However, there are many non-closed loop system activities that occur while operating the Regen ECLSS systems.

UPA contains a Recycled Filter Tank Assembly (RFTA) which collects concentrated brine as the UPA processes pretreated urine into distillate. The RFTA was designed to accommodate $85 \%$ reclamation of the pretreated urine before requiring to be changed out, which was planned to occur once a month. As the filter does require a change out, it therefore requires the flight control team to plan crew time to perform this activity, thus breaking the closed loop system. 
The WPA's WW tank can hold up to 100lbs of waste (condensate \& distillate) as well as its WS tank and water delivery accumulator that can hold up $125 \mathrm{lbs}$ and $20 \mathrm{lbs}$ of potable water, respectively. It was expected that the USOS would collect $70 \%$ of the ISS condensate and produce an excess of water ${ }^{1}$, thus requiring crew to offload water from the water storage tank into CWC-Is. By requiring crew to offload the WPA tanks into CWC-Is, the closed loop system is broken.

The rest of the systems within the Regen ECLSS systems were designed to be more closed-loop, with the only need for crew intervention being preventive maintenance of limited life items, known as Orbital Replacement Units (ORUs), as their certified life span expired.

These ORU life spans are on the order of years and therefore the planning of crew time required to perform these activities have minimal impact on the closed-loop system operation.

Due to various reasons described above as to why the Regen ECLSS is not a truly closed-loop system, the ISS ECLSS flight control team determined that they would need to begin managing water not only as a long term consumable, but as a short term constantly changing system. Therefore, the flight control team began the ongoing task known as "water balance" to comply with the numerous (and ever changing) hardware and operational constraints levied on the system by the engineering and safety communities.

The water balance is managed via a Microsoft Excel spreadsheet which allows the on-console ECLSS flight controller to update a variety of parameters. The spreadsheet predicts the outcome of overall water balance, reference Figure 2.

As seen in Figure 2, there are a number of inputs the flight controller makes as a data point and which is then used by Excel functions to predict future water operation needs.

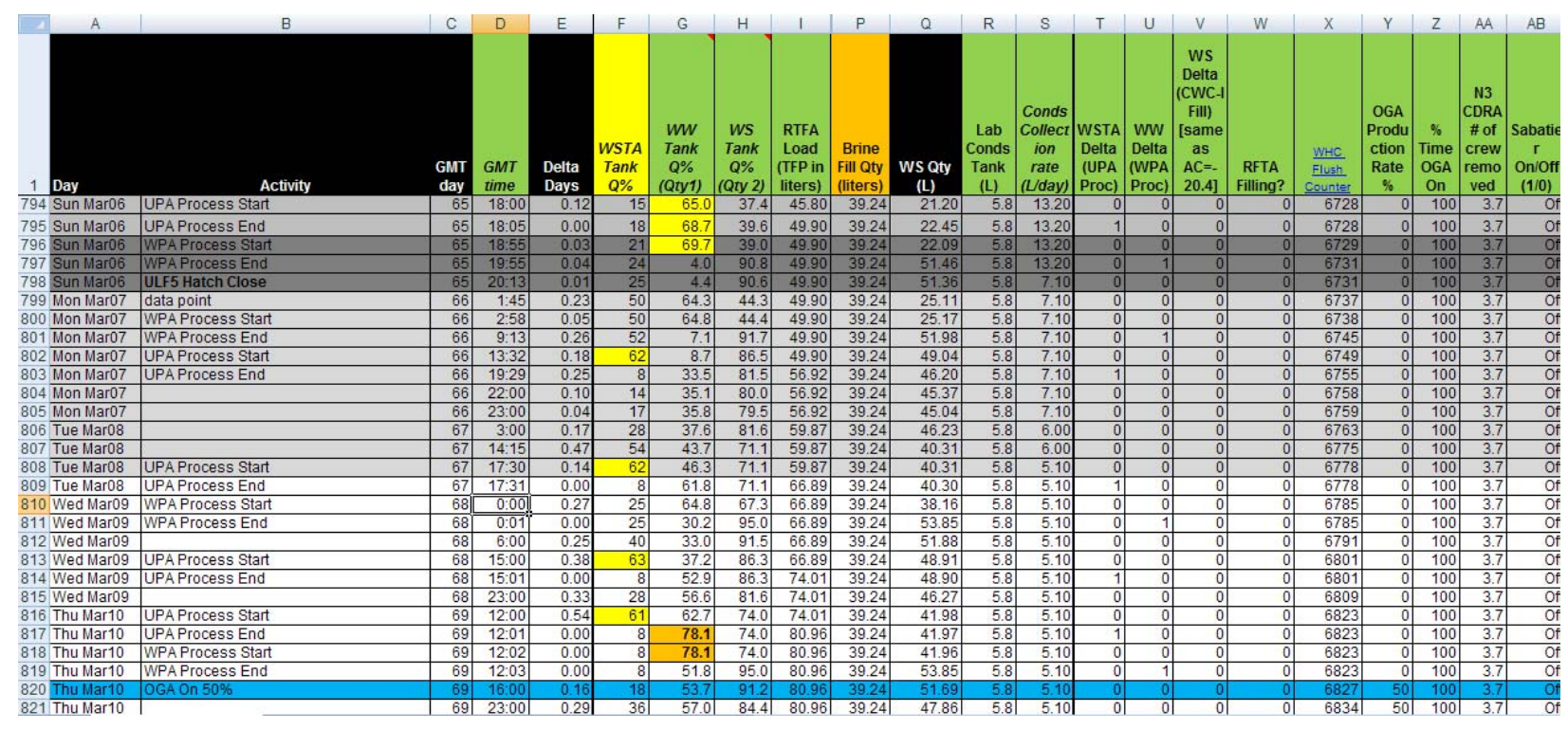




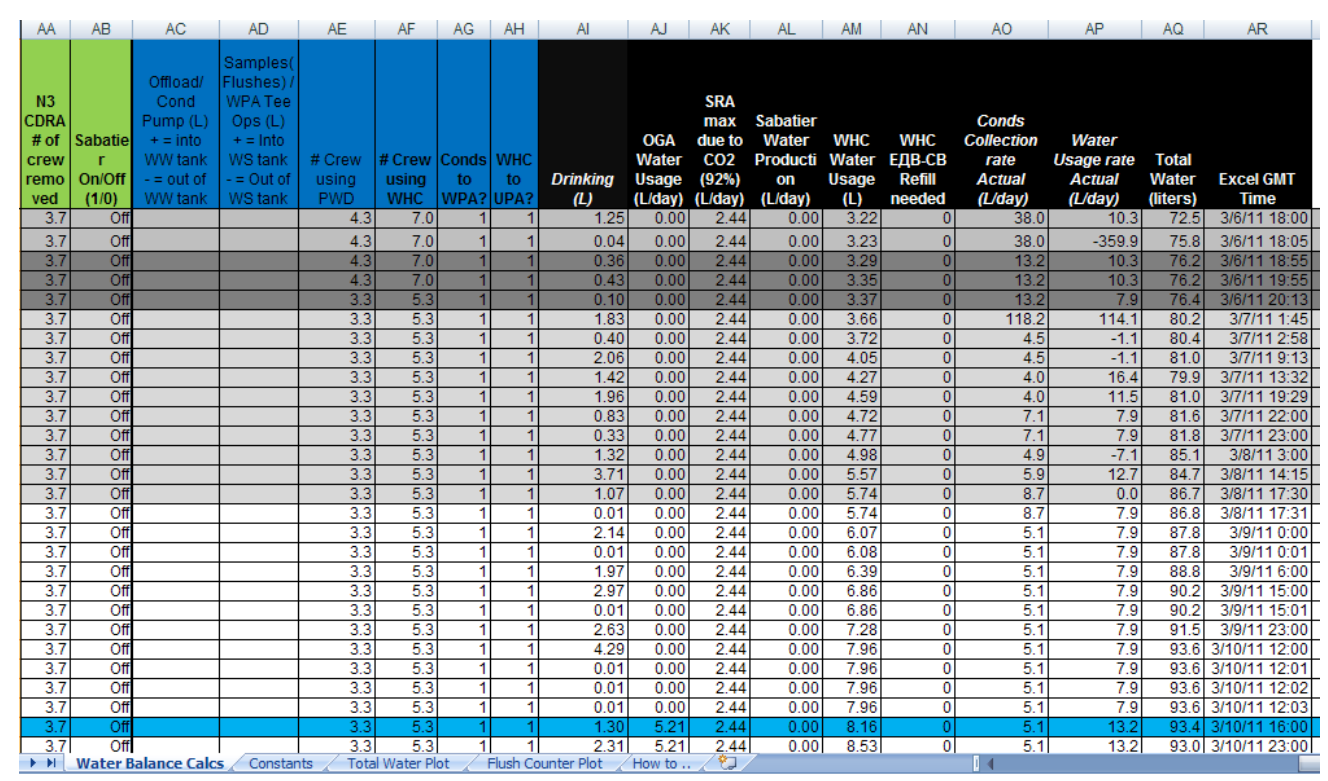

Figure 2: Water Balance Spreadsheet

These inputs are:

GMT - The current time when inserting a data point or when looking ahead at an upcoming event.

WSTA Tank Q\% - The quantity in the UPA's urine tank. When the tank reaches 70\%, the UPA begins a process cycle.

WPA WW Tank Q\% - The quantity in the WPA's waste water tank. When the tank reaches $65 \%$, the WPA begins a process cycle. The flight control team frequently commands WPA between software states that will allow more control of its processing time, preventing it from automatically processing at this $65 \%$ level.

WPA WS Tank Q\% - The quantity in the WPA's water storage tank. WPA won't process automatically if this tank is greater than $80 \%$, in order to avoid short processing cycles.

RFTA Load (TFP) - The amount of fluid throughput in the RFTA. The maximum possible at $70 \%$ recovery is 95.2L (210lbm).

Brine Fill Qty - The amount of pretreated urine used to fill the RFTA upon initial installation. This is required as the RFTA initially flies at vacuum and is filled on-orbit prior to its installation for urine processing.

Lab Condensate Tank Qty - The quantity in the Lab condensate tank.

Condensate Collection Rate - The daily rate of condensate collection.

UPA Process - If the WSTA Q\% value reaches $70 \%$, the user inserts a "1" in this field and that causes the spreadsheet to "run a UPA process", resetting the WSTA Q\% to $8 \%$ and adding the amount of processed fluid to the WPA WW tank Qty cell.

WPA process - If the WPA WW Qty value reaches $65 \%$, the user inserts a " 1 " in this field and that causes the spreadsheet to "run a WPA process". The spreadsheet resets the WW tank qty to $4 \%$ and moves the fluid amount into the WS tank qty cell. However, if that would cause the WS tank qty cell to max out (no greater than 95\%), then WS qty cell is set to $95 \%$ and the WW tank qty cell is reduced by only the amount of fluid that was moved to the WS tank cell. 
CWC-I Fill - If the user inserts a " 1 " in this cell, the spreadsheet offloads 18 liters from the WPA WS tank, as this mimics crew filling a CWC-I with WPA product water.

RFTA Filling - When an RFTA is first installed, it is at vacuum and requires 40 liters of pretreated urine to fill it to reach ambient pressure. When the user inserts a " 1 " into this field, the WSTA qty tab resets to 5\% and the Brine Fill Qty column increases, but no water is added to the WPA WW Tank Q\% cell.

WHC Flush Counter - This value is a number on the WHC that tracks the number of flushes and is called down periodically by the crew. The spreadsheet uses this value to predict when the next required fill of the ЕДВ-СВ will be needed. Upon this fill being required, the spreadsheet will automatically set the "WHC ЕДВ-CB Refill Needed" column to 1 and the WPA WS tank qty is reduced by however much is listed in the "WHC Water Usage" column, which is the predicted amount of fluid required to refill the ЕДВ-СВ.

OGA Production - The OGA operates between $22-100 \%$ production rate, or may be in a standby mode which equates to a $2 \%$ mode.

OGA On Time \% - This is the amount of the day in which the OGA is processing. There is an option for the OGA to operate based on the ISS being in a day (insolation) pass only, in order to save power on the ISS batteries. This cell, along with the OGA Production cell, is used internally to calculate a water usage rate by the OGA, and that value is removed from the WPA WS tank qty cell.

Node 3 CDRA \# of Crew Removal - This cell is used to help calculate the amount of water the Sabatier creates. There are $3 \mathrm{CO} 2$ removal systems onboard the ISS and therefore the Node 3 CDRA does not remove all 100\% of crew $\mathrm{CO} 2$ waste. Therefore, the amount of crew removal is required as an input. This value is based on past flight experience and the current operating status/efficiency of the Node 3 CDRA, Lab CDRA, and Vozdukh CO2 removal systems.

Sabatier On/Off - User input to the current state of the Sabatier.

WPA WW Tank input/output - It is possible that crew can pump in extra condensate from a CWC or ЕДВ into the WPA WW tank. Crew can also offload the tank if required. This cell is used to adjust any manual input in or out of the WPA WW tank.

WPA WS Tank input/output - Similar to the above item, this cell is used to manually input water in or out of the WS tank. If crew fills a CWC-I from the WS tank, or a variety of samples are taken, or a manual fill of the ЕДВ-СВ is needed, this cell can be used to model those activities. It is also possible to connect a CWC-I to the WS tank and transfer water from the CWC-I to the WS tank in order to insure there is enough required water in the potable bus for nominal operations.

\# of Crew using the PWD - This value is used by internal calculations to determine the amount of crew drinking rates that is removed from the WPA WS tank qty cell.

\# of Crew using WHC - This value is used by internal calculations to determine the amount of urine being inputted into the UPA WSTA qty cell.

Condensate to WPA - There are occasions when the WPA cannot accept water, in which case the crew swaps a variety of jumpers to route condensate collection into the Lab Condensate tank, rather than into the WPA WW tank. A user input of " 0 " in this cell stops any condensate collection from being added to the WPA WW tank qty cell.

WHC to UPA - There are occasions when the UPA cannot accept urine, in which case the crew configures the WНС to input urine into an internal ЕДВ-У, rather than into the UPA WSTA. A user input of " 0 " in this cell stops any urine collection from being added to the UPA WSTA qty cell.

Other Columns - There are a number of other columns shown in Figure 2. These are used by the excel sheet for internal calculations and/or used by system experts within the flight control group for reasons beyond the scope of on console Water Balance operations. 


\section{Challenges to Water Balance}

As stated before, the Regen ECLSS systems were designed based on spec rates and even then required crew interface. Although the long term crew rates can be shown to match documented spec rates, short term rates can vary. In addition, there are a variety of new operational constraints that have been put in place due to various failures the Regen systems have experienced. All these, along with other generic ISS operations, require a significant amount of water balance management.

The Regen ECLSS systems require a number of out-of-the-loop equipment to operate. This includes CWC-Is in which to store water, ЕДВ- $\mathrm{Y}_{\mathrm{S}}$ to store pretreated urine, periodic replacement of RFTAs, and removal and replacement of ORUs. Given that at any time during ISS operations not all Regen ECLSS systems may be readily available, modifications to the amount of fluid processed for recovery versus what is transferred to Progress Rodnik tanks for disposal changes, thus affecting the "closed-loop" water balance system.

System failures occur. Any time that any part of the Regen ECLSS systems fails, an input or output rates can change. This also affects the water balance plan. For example, if the UPA were to have a failure, $\sim 4.5$ liters each day is no longer being supplied to the WPA for processing. This has a significant effect on water balance and requires replanning of the overall system to insure crew water needs are met. If a failure occurs in the Russian water systems, the US systems are available for cosmonaut usage (as is the Russian system available for astronaut usage if a failure occurs on the USOS). The extra crewmembers now tied into the system causes a significant change to the overall water balance, as this can change the input/output rates from $\sim 3-7$ liters a day.

A number of failures have occurred in the Regen ECLSS systems and other water systems that have added operational constraints and thus added to the complexity of managing the water balance.

In late 2009, early 2010, indications of clogging was seen in the WPA waste water lines. It was discovered that biosludge was forming in the WPA WW tank, and when broken free, began clogging various valves and lines within the system. It is believed that the sludge builds and is trapped between the bellows' convolutes in the tank, and when the tank is filled to high levels, the sludge is allowed to release as the bellows flexes to its expanded position. As a constraint to preclude the release of sludge, processing of the WPA was, until very recently, limited to waste water tank quantities between $55-75 \%$. This is done in order to ensure constant cycling of the bellows around a designated quantity and thus minimizes the release of sludge. This has had a major impact to overall planning as it has removed operational flexibility from processing when the tank has 0-95 lbs of water, to now only being able to process if the tank has 55-75 lbs of water. Although, if crew requires the water to be processed sooner, a short cycle (processing when the quantity is less than $55 \%$ ) is allowed, but discouraged. (Note: At the time of this paper being submitted, this constraint was changed to allow for a larger range, but still requires intensive ground operations to meet the new requirements.)

Upon initial activation onboard, the UPA's Distillation Assembly (DA) experienced a number of issues due to physical interference. Upon further investigation, the engineering teams have determined that thermal expansion may play into the cause of this failure. This has led to operational constraint on run time and cool down times. In order to ensure that the DA does not flood with excess water, it is also desirable to allow heater temps to reach a steady state value before stopping a process. Given all the constraints, the UPA cannot run more than 7.5 hours at one time and requires a 5 hour cool down between each run. In addition, it is desirable to not run less than 4 hours. Nominally these constraints are not an issue with 3-4 crew nominally inputting into the UPA, however, they are far from the "closed-loop" system the Regen ECLSS system was originally planned to be.

As the RFTAs are launched at vacuum, they require a filling of $\sim 40$ liters of pretreated urine before UPA can process any urine into distillate. As designed, the RFTA would be installed and the system would simply backflow into the RFTA and once it was full, the system would then begin processing urine. However, it was found that onboard, the vacuum force pulling the pretreated urine into the RFTA was causing the pretreated urine to foam at the outlet filter of the RFTA (inlet filter when filling via the output port). As a result, crew is required to mate a bypass jumper to allow the urine to fill the RFTA via the nominal input port. This adds a challenge to the water balance plan as this activity's planning has to be predicted. If the predicts are not correct, then processing of urine 
does not occur when planned and potentially some of the fluid may be lost. This is due to the WHC being configured to output to an internal ЕДВ or crew uses the Russian АСУ, until the hose configuration is made, thus, that urine may not be made available for reclamation.

Similarly, the number of times an RFTA has to be changed out has increased as well. It was found that particulates come out of solution within the UPA earlier than predicted. This led to clogging of a DA and required a complete change out of the DA and Fluid Control and Pressure Assembly (FCPA) ORUs. In order to avoid this from occurring again, the reclamation ability of an RFTA was reduced from $85 \%$ to $70 \%$. This means that the amount of fluid that can pass through the RFTA before a change out is required was reduced by $1 / 2$. As predictions and timeline planning is required for these change outs, any error between predicts and actuals allow for potential loss of urine. In addition, an RFTA requires 40 liters of fluid to fill it from its launch config, being at vacuum, to being full. This filling process takes $\sim 26$ crew days ( 1 week for $3 \mathrm{crew}$ ) and during that time, urine is not being processed, so distillate is not being sent to WPA. This lack of distillate is another factor in the water balance challenge.

A number of issues have been seen with the CWC-Is as well as their interface to the Regen ECLSS systems. Due to ground processing, which involves pulling vacuum on the CWC bladders, air permeates into the bag. Unfortunately, the users of the potable bus have little tolerance for free gas. Therefore, the CWC-Is needs to be degassed prior to being inputted into the WPA water storage tank. This requires $\sim 1$ hour of crew time per CWC-I, tracking of the bag degas status by MCC-H, and additional planning prior to supplementing the potable bus.

The water in the CWC-Is do have a shelf life and when it is near the expiration date, they need to be emptied into the water system. This requires that the water system needs to be able to accommodate the water prior to expiration. This takes advanced planning and sometimes requires pumping in of the water followed by an offload of the system. The lifetime of the on orbit iodinated water is a moving target based on ongoing ground testing. This adds to the complexity of prioritizing use of these bags since a CWC-I that may have expired one month might not be expired next month based upon lifetime ground test results. This moving timeframe in which the bag is "unexpired" may be as short as a week.

Both CWCs and CWC-Iodines have developed leaks both while stowed and while being filled on orbit. These leaks drive the need to empty these containers into the system to save that water. If the leak is a pinhole bladder leak, operationally there is not a constraint as to when the water must be transferred, however, if the leak is a larger leak, it has been found that the water begins to grow bacteria and smell in about 1-2 weeks. In order not to expose crew to these smells, this drives the ground teams to determine how to incorporate an unplanned $\sim 22$ liters of water into the system in a timely manner. Leaking bags that are not (or cannot) be emptied back into the system must be double bagged and subsequently disposed of on ATV, Progress, or other vehicles, resulting in loss the CWC hardware and any water not reclaimed. CWCs and CWC-Is also have a limit to the number of fill/drain and QD mate cycles which must be tracked by ground personnel.

CWCs do not have quantity indicators, therefore, when the container is filled and subsequently used, the ground controllers rely on the crew to determine how much fluid is left in the bag. This is not a very precise or accurate measurement. This uncertainty increases the risk of overfilling the CWCs and results in the need to purposefully underfill them. Underfilling the bags is necessary to prevent damage and leakage to the CWCs but means less available space to store water.

Lastly, the major impact to water balance is that of actual rates. Spec rates have been shown to be very accurate over a long period of time over multiple years of onboard history. However, actual rates over a short term change drastically. A number of variables affect these actual rates. Individual crew metabolic is one of the major factors. There have been cases where some crews urinate more often than others, or some may deposit large amounts of urine at each use. Deposits have ranged from $50 \mathrm{~mL}$ to $600 \mathrm{~mL}$, thus making accurate predictions difficult. Crew location also factors into this. With a crew of six onboard the ISS, their locations during the work day, during meals, and during sleep hours change. On average, the crew is split $3 \& 3$ between using the USOS and Russian toilets, however, there have been times when over the course of a week, telemetry has indicated the WHC was being used by 4-5 crew members. As micromanaging crews' toilet use is generally avoided by the flight control team, these significant changes have affects on the overall water balance plan. 
Crew condensate collection is highly variable based upon individual crew perspiration and drinking habits. The US collection rate is also highly dependent upon the modes of the various condensing heat exchangers onboard ISS. Russian CKB (condensing heat exchangers) operate intermittently based upon air temperatures in the SM, which are a strong function of ISS attitude and solar beta angle. US CCAA condensing heat exchangers must go through monthly dryout cycles to protect against microbial growth. MCC-H adjusts Low Temperature Loop (LTL) coolant temperatures and flow rates to affect these dry outs. Additionally, the CDRA CO2 removal efficiency is a strong function of coolant loop temperature, and LTL temperatures are lowered when additional CO2 removal capability is required onboard, most notably during high crew loading during Shuttle docked missions or direct crew handovers. Additionally Inter-module ventilation (IMV) factors into the water balance as well. Over time the IMV fans become dirty and degrades flow between modules. This affects where the condensate is collected, USOS vs. Russian segment, and thus affects water balance.

In addition to all the previously stated challenges, a nominal Regen ECLSS system still requires significant water balance operations. As MCC-H experience shows that the overall water system on ISS would benefit from more system capacity. The nominal ISS planning template is a week out, and changes to crew timelines should be minimal within 4 days of execution. However, the capacity in storage tanks that do not require crew action is limited.

The WPA waste water tank has a total capacity of $100 \mathrm{lbs}$ of water mixed between distillate and condensate. Until recently, working tank preferred upper limit was limited to $65 \mathrm{lbs}$ of water due to concerns with biofouling in downstream components. The tank minimum volume is $4 \mathrm{lbs}$, meaning the working tank volume is $61 \mathrm{lbs}$. Assuming observed condensate and urine collection rates, the time to fill the waste water tank is on the order of 2 days. If one assumes the tank is likely at its midpoint at any random point in time, the time to respond is on the order of 1 day. These response times would be even less if Sabatier is fully operational, US begins processing RS urine, or the RS CKB are deactivated. Any WPA failure, or the nominal case where the WPA water storage is full due to a large net positive water balance, will result in MCC-H having to take immediate action to potentially increase the response time, and ultimately result in crew time being spent to offload the tank.

The water storage tank has 115 lbs of working volume. The WHC flush ЕДВ autofills when the ЕДВ is empty, meaning MCC-H must protect 22 liters (49 lbs) for this fill at all times, or add to the operational complexity of the water balance by attempting to track the quantity in the flush ЕДВ. There is no quantity indicator on this ЕДВ, and the quantity can only be estimated by a flush counter that must be reported manually by the crew. 3 US crew will nominally use $18 \#$ per day from the Potable Water bus, meaning the time to drain the tank is $\sim 4$ days. If one assumes the tank is nominally at its midpoint at any random point in time, the time to effect is $\sim 2$ days. Once OGA is active, these times will only shorten.

The WSTA urine storage tank has a nominal working volume of $\sim 18 \mathrm{lbs}$. The time for three crew to fill the volume is $\sim 2$ days, and $\sim 1$ day from midpoint to tank upper limit. If urine processing is not possible, for example the RFTA is full or there is a UPA failure, crew must take action to swap WНС to internal ЕДВ-У. Wetting the ЕДВ-У results in loss of a consumable, since the ЕДВ-У has a 90 day wetted lifetime.

Given all of the above, MCC-H has scheduled twice weekly, one hour long (two crew hours per week) "water balance" activities to compensate for the relative lack of capacitance in the water system.

\section{Conclusion}

Since November of 2008, Regen ECLSS systems have been operating onboard the ISS and providing a near closedloop system for human waste and metabolic needs. The systems were originally designed based on a certain set of assumptions with regard to metabolic and system rates. Although these assumptions may be accurate assumptions over a long period of time, they are not accurate in the short term. Due to a variety of factors, ranging from crew location and metabolic to system efficiency, the actual rates vary and change every few days. Due to a variety of unforeseen failures within the Regen ECLSS systems, numerous operational constraints and extra human intervention is required to operate the systems. Due to this, the system has become an under-damped system and thus requires daily water balance management to insure that the water quantity in the various sub-systems are within 
certain levels as well as maintaining an ample supply of potable water for crew drinking and hygiene. Due to the ongoing changes in rates, water balance can only be predicted accurately for approximately 5-7 days of operations and semi-accurately for 2 weeks into the future. With all these variables, "Water Balance" has become one of the most challenging daily operations the ECLSS flight control team has to handle both on and off console.

In addition, a lesson learned from onboard experience has shown that future systems should be sized for more time to effect, or the crew time required to respond should be included in ops concepts up front.

\section{References}

1. D.A. Yeoman, B. Shkedi, and B. Tobias, ISS Water Architecture and Operational Plan, SAE International Technical Paper: 08ICES-0123, 38 ${ }^{\text {th }}$ International Conference on Environmental Systems, June 30-July 3, San Francisco, CA 